\title{
Exploring Tracking Needs and Practices of Recreational Athletes
}

\author{
Maulishree Pandey, Michael Nebeling, Sun Young Park, Steve Oney \\ University of Michigan \\ Ann Arbor, USA \\ (maupande,nebeling,sunypark,soney)@umich.edu
}

\begin{abstract}
Sports are the primary physical activity for over 52 million people in the United States, a vast majority of which are recreational athletes. The general tracking practices and needs of this population have not been studied. In this paper, we explore how recreational athletes use tracking technologies to track their sports performance. We conducted interviews with 25 recreational athletes that are runners, soccer, tennis, and basketball players. We found our participants engaged in supplementary physical activities like exercise and strength training to improve their sports performance. They used wearables and mobile applications to track general physical activity data. However, they were unable to track sport-specific techniques due to limitations of tracking technologies, and desired better tracking support for the same. We present design opportunities for future personal informatics tools to better support the needs of recreational athletes.
\end{abstract}

\section{CCS CONCEPTS}

- Human-centered computing $\rightarrow$ Ubiquitous and mobile computing systems and tools.

\section{KEYWORDS}

Personal informatics, activity tracking, recreational sports

\section{INTRODUCTION}

Sports is one of the many ways in which people seek to improve and maintain their health and fitness. In the United States alone, sports are the primary physical activity for over 52 million people [15]. The vast majority of these are recreational athletes. We define recreational athletes as people who frequently play a sport with a goal other than material compensation. These goals could typically include playing for fun, to improve health, or as a way to socialize.

Personal informatics tools like activity trackers and mobile applications are designed to track metrics such as steps, distance and heart rate. These are important to give users a general sense of their physical activity. They have shown promise in improving people's health [16]. However, they are of limited utility when one wishes to track detailed information on more complex activities such as exercise and recreational sports.

Permission to make digital or hard copies of all or part of this work for personal or classroom use is granted without fee provided that copies are not made or distributed for profit or commercial advantage and that copies bear this notice and the full citation on the first page. Copyrights for components of this work owned by others than the author(s) must be honored. Abstracting with credit is permitted. To copy otherwise, or republish, to post on servers or to redistribute to lists, requires prior specific permission and/or a fee. Request permissions from permissions@acm.org.

Woodstock '18, fune 03-05, 2018, Woodstock, NY

(c) 2018 Copyright held by the owner/author(s). Publication rights licensed to ACM. ACM ISBN 978-1-4503-9999-9/18/06 . \$ \$15.00

https://doi.org/10.1145/1122445.1122456
Previous HCI research has designed tracking technologies for individual sports and techniques. These are useful technical contributions for engineering tracking tools. However, they do not inform us about how recreational athletes generally use tracking technologies. Given their sizable population and the unique nature of sports as a physical activity, studying their needs and practices can offer useful insights for design of personal informatics systems.

We have conducted interviews with 25 recreational athletes across four different sports of running, soccer, tennis and basketball. We chose these sports because they are among the most popular for recreational athletes [1]. Grounded in interview findings, this paper makes following contributions:

1. A better understanding of how recreational athletes engage in supplementary physical activities to improve their sports performance, and their use of general tracking tools and workarounds to track their sport and physical activities.

2. An analysis of measures recreational athletes desire better tracking support for, which we describe as having a "long tail."

3. Design opportunities for personal informatics systems to better meet the needs of recreational athletes.

\section{RELATED WORK}

\section{Personal Informatics Tool Use}

Li et al. [18] defined personal informatics systems as tools that enabled people to collect relevant information about self and helped in insightful reflection. They developed a linear stage-based model of personal informatics systems: preparation, collection, integration, reflection, and action. Epstein et al. [10] recommended extensions to this model by looking at behaviors before the preparation stage, and after tracking had been stopped. They found that motivations to begin tracking could be quite diverse. Similarly, Rooksby et al [24] studied usage of health and wellness tracking technologies, and found that people's tracking requirements could be complex. For certain extreme users, termed 'Quantified Selfers', tracking needs could be varied enough for them to design their own custom tracking tools [5]. Other studies have looked into long-term usage of fitness trackers [11], why people abandon tracking [6, 9, 17], and how wearability of tracking tools can be improved [12, 23]. However, these are broad studies and have have addressed general health and wellness tracking.

\section{Recreational Sports and HCI}

The HCI community has proposed solutions to increase the visibility of physical activity $[7,8]$, and encouraged users to be more active through positive social reinforcement $[8,19]$. Researchers have also evaluated tracking systems specific to sports [3, 14, 20, 21, 25]. These studies are useful in providing an understanding of how specific metrics matter in individual sports. More recently, researchers have 
conducted qualitative studies with recreational and professional athletes. Interviews with professional tennis players have revealed that they desire more 'specialized' information [13]. Tholander and Nylander interviewed endurance sports' athletes, runners and golfers about their use of wearables [22,26]. They found that athletes focused on their "feelings" to evaluate their performance, and wanted tracking tools to reflect this internal assessment. However, none of the studies inform us of general usage, needs and challenges of recreational athletes with activity tracking tools. We wish to fill this gap through our study.

\section{STUDY}

We conducted semi-structured interviews with 25 recreational athletes ( 7 female, 18 male) across four sports: running (8 participants), basketball (6), soccer (5), tennis (5), and 1 participant in both soccer and tennis (1). Interviewees were recruited from recreational sports leagues and online sports forums via emails, flyers, and social media postings. Their ages ranged from 19 to 48 (mean: 30, median: 27).

Interviews were structured into three parts with questions around participants' motivations and goals in playing sports, their usage of tracking tools, challenges with tracking tools, and any wishes they had for future technology to better fit their sports. Each Interview lasted 25-40 minutes, and took place via Skype, or in person. Participants were compensated with $\$ 10$ Amazon gift cards. All interviews were audio recorded and transcribed for data analysis. Five researchers conducted an affinity analysis to identify key themes.

\section{RESULTS}

This section details the findings from the interviews. Participants' quotes are abbreviated by sport: running $(\mathrm{Rx})$, basketball (Bx), soccer (Sx), and tennis (Tx).

\section{Engaging in Sport and Supplementary Activities}

Participants played their sport 2-3 times each week. They had different goals for playing the sport like staying fit, gaining exercise, having fun, and enjoying with others. They also enjoyed the competitive aspect of sports, and strove to better their own performance: "In that perspective, improvement is being able to run a little bit further than I did the day before. As long as I get a little bit further... For me it is the chase of getting a little bit further" (R4).

Participants believed their sports performance was a combination of skills needed to play the sport and general fitness levels. Thus, they practiced certain techniques to perfect it: "I care about my jump shot the most. I want to improve my accuracy at shooting mid-range shots... I practice that more than anything” (B3). We noted individual differences among techniques that participants practiced. These were due to their prior experience at the sport and existing skill with techniques. To improve fitness levels, participants undertook activities like cardio, strength training, and healthy eating. They planned their workout around the sport: "To play soccer... I actually run during the weekday... for weekend soccer. For both sports (soccer and tennis), I train my legs more than the upper body. I don't do weights much. I try to run regularly... I care about the leg muscles." (S6/T6). Participants were motivated to improve at these supplementary activities because they believed it impacted their overall sports performance: "I use my Apple Watch for practice and stuff... Which I think is directly correlated to my performance because if I look and say, 'well I ran for four miles and it has been $20 \mathrm{~min}$, (so) I ran faster,' that is something specifically toward the running component of soccer" (S1). Subsequently, improvement in overall performance helped them enjoy the sport more: "One of the ways I have fun is by playing better... Like you know just being a better basketball player." (B4).

\section{Usage of Tracking Tools and Technologies}

Participants reported tracking using wearables, mobile applications, and even repurposing mobile applications not intended for health and fitness. None of the participants playing soccer, tennis and basketball used devices tailored to their sport. Instead, they used general-tracking devices from brands like Garmin, FitBit, Apple.

All runners in the study used either wearables or mobile applications. They were largely satisfied with the data collected by the technology but expressed concerns about the accuracy. Participants of other sports used wearable devices and mobile applications indirectly for their sport. They tracked measures such as distance, steps, and speed during practice and friendly matches. They acknowledged the inaccuracy of data but unlike runners did not view it as a big disadvantage. We believe this difference could be because most existing trackers track measures that can be easily incorporated by runners in performance improvement, but not so much by other athletes. However, participants of other sports mentioned they desired better recognition of their sports. One participant said his activity tracker would occasionally characterize the sport as 'exercise' or 'running' instead of 'sport', and vice versa. Another soccer participant, who used an older FitBit, said he had no option of selecting his sport within the FitBit mobile app. His data during matches was collected as 'running' activity. Participants felt this mischaracterization impacted the data on calories, distance ran, etc.

Athletes also used applications not designed for tracking physical activity. Two participants used Google Maps to measure the distance of their running routes. Another participant used a note-taking application on their phone to note down the details of his workout (e.g., exercises carried out, number of repetitions). This helped him plan his workout. Similarly, tennis players used mobile cameras to record their forms and posture: "Casually I sometimes put my phone down... I hit a couple serves and see where I am. I look at the speed... I look at my form during serve." (T4). However, participants faced issues with these workarounds. One tennis participant mentioned he faced difficulty in analyzing his form because of video angles. Participants also struggled to find someone to record their video.

Lastly, participants reported referring to their feelings (e.g., feeling of competency, strength) and to the memory of mentally tracked measures (e.g., number of shots, passes). This was especially true for the four participants who were not using any tracking tools at the time of our study. They felt existing technologies could not track such measures: "I've never seen anyone who's using a gadget for tennis, except the Fitbit thing but that's only for pedometer or heart rate for fitness. It does not help me for tennis" (T3).

\section{Desired Metrics and Categories}

We inquired about measures participants wanted better tracking support for. This revealed a variety of unmet tracking desires. We 
refer to this as a 'long tail' of desired metrics because of the individual differences in tracking desires of participants, within a sport as well as across sports. We recorded 70 unique measures (table 1), categorized into four groups.

The category of 'technique' includes skills specific to the sport. We found that technique information, despite being most desired, was least supported by tracking tools. Most of these measures were mentioned by non-runners: "I would like to know how fast my shots are or my racquet speed... I think they are critical to track especially in a serve, (because) speed trumps all. I think speed is my primary focus." (T5). Participants in these sports felt less supported by existing tracking technologies.

The second most popular categories were 'physical status' and 'outcome'. The former includes measures on physiological state of athletes. Participants in all sports wanted further statistics like respiratory rate, blood pressure, muscles' output, etc. The category of "outcome" included measures on game statistics like wins, losses, and individual scores. Participants playing tennis, basketball, and soccer mentioned they mentally tracked these measures. Reflecting upon these numbers allowed them to conclude if they had improved or not: "You kind of keep the mental record for yourself like 'Oh, I made that many points in that game, that was good'." (B5). However, counting mentally was tedious, and participants wished for a technology to compile and present this data.

"Social support" refers to data that can be utilized to improve team performances. Only one soccer participant explicitly stated she wished to track "team connectedness", as she felt the outcome was better when the players played as a close-knit team. Other participants mentioned they only mentally tracked others' performances during a match to make decisions such as whom to pass the ball, how to change their play, etc.

\section{Need for Appropriate Device Placement}

Participants elaborated on placement limitations of tracking tools. Participants who owned wearables had to wear it on their wrists, while those who used mobile applications had to carry the phones. This posed problems to tracking for soccer and basketball athletes who mentioned that sports' rules didn't allow them to wear anything on their wrists and arms as it posed risk of injury. Further, carrying a phone hindered sprinting and running in all sports. Therefore, participants refrained from tracking during matches. Further inquiry revealed that athletes wanted to be able to wear trackers at unobtrusive locations so that it would not get in the way of play. Thus, runners and tennis players preferred wrists, basketball players preferred ankles, and soccer players preferred chests. Participants acknowledged that wearing it on body parts not used in the sport may affect data quality, but they would prefer a "less accurate" tracker over "an accurate but obtrusive" device. We also found individual differences in preferences. For example, one runner wanted to wear the tracker on his wrist to check statistics while running. Another runner wanted to wear the tracker on his ankle to avoid being distracted with data mid-run.

\section{DESIGN OPPORTUNITIES}

Based on the results, we discuss two major design opportunities for personal informatics systems to better support recreational athletes.
Table 1: Desirable metrics by type (70). The numbers in parentheses are the number of participants who asked for a given metric for their sport.

\begin{tabular}{|l|l|}
\hline Type & Metrics \\
\hline Technique & $\begin{array}{l}\text { How hard the ball was hit (5), Movement (direction, } \\
\text { area) (5), Ball spin (3), Ball location (3), Ball speed (3), } \\
\text { Live pace performance (3), Shot attempts (1), Angle } \\
\text { of shot (1), Person defending (1), Shooting accuracy } \\
\text { (1), Distance from the hoop (1), Ball release time } \\
\text { (1), Whether shot was contested (1), Vertical jump } \\
\text { distance (1), Potential moves/poses to make (1), Ball } \\
\text { height (1), Serve trend/tendency (1), Overall presence } \\
\text { on the floor affecting the score of the game (1), Steps } \\
\text { per time period (1), Rebounds (1), Assists (1) }\end{array}$ \\
\hline $\begin{array}{l}\text { Physical } \\
\text { Status }\end{array}$ & $\begin{array}{l}\text { Respiratory rate (3), Cardio performance (3), Speed } \\
\text { during the play (3), Body change (muscles output) } \\
\text { (2), Blood pressure (2), Metabolism rate (1), Postures } \\
\text { (1), Sprints (1), Calories burning (1), Strengths \& } \\
\text { weaknesses (1) }\end{array}$ \\
\hline Outcome & $\begin{array}{l}\text { Progress (4), Agility (2), Distance (2), How many } \\
\text { points you gave up (2), Goals you made (2), Game } \\
\text { history (2) }\end{array}$ \\
\hline Team & Team Connectedness (1) \\
\hline
\end{tabular}

\section{Tracking Support for Multiple Activities}

Our interviews revealed that recreational athletes engaged in supplementary physical activities to improve their overall performance. They often used activity trackers to monitor exercise related activities, and assess their improvement at these activities. However, they were unable to effectively track sports-specific techniques, especially in soccer, tennis and basketball. We therefore recommend that activity trackers should be enhanced to track multiple activities, without forgoing the support for general data. Our recommendation builds on prior literature that has found that tracking technologies currently collect generic data [2], despite users wanting to track multiple activities [5].

Enable Combining Data with Multiple Sensors. Most wearables are designed with limited number of sensors, and can track select metrics. It is not possible for a single sensor to track many of the metrics that athletes want to track in conjunction. Bearing these in mind, we recommend that devices, sensors, and applications should be designed to exist as part of a larger, uniform tracking system. Thus, designers should consider how a new sensor or wearable can extend the capabilities of other, perhaps even, older sensors, and make the overall system more versatile and "holistic".

Provide Placement Options For Tracking Technologies. Most wearables are designed to be worn on wrists [4]. However our interviews showed that recreational athletes did not always wish to wear the tracking technology on their wrists. This need was driven by sports rules, other physical activities they undertook, and personal preferences. To adequately support athletes in tracking multiple activities, these reasons need to be taken into account. Activity trackers should have multiple placement options. Designers should examine sports rules when designing wearables, as athletes may 
not be permitted to wear trackers at certain body locations for fear of injury. It should be possible to place the wearable at unobtrusive positions. For example, during a soccer or basketball game, athletes should have an option to wear the tracker on their chest or ankles respectively. But during exercises or running, the same tracker should have the option to be worn at convenient locations such as wrists or arms to allow the user to access data quickly. Our recommendation builds on previous HCI research to improve wearability of activity trackers [12, 23].

\section{Allow Self-Defined Goals}

Our study demonstrates that recreational athletes worked on improving their performance. Getting better allowed them to enjoy their sport more. They therefore tracked measures that mattered to them, and desired tracking support for related metrics. We found individual differences in the desirable metrics of athletes. Even athletes playing the same sport had different goals for their improvement, and this drove different tracking requirements. Given this variety in athletes' needs, we suggest that personal informatics systems should enable athletes to define and articulate their own goals. Depending on the goal stated, the technologies should provide suggestions to athletes about how they can best track measures related to their goals. These suggestions could include various activities an athlete should undertake, and their execution on the field. This builds on Choe et al's [5] recommendation that technologies should initially suggest the measures one should track. This will greatly reduce the burden on recreational athletes to collect data, implement changes, and reflect upon their sports performance.

\section{LIMIATIONS AND FUTURE WORK}

We recognize that our sample is male-dominant. Thus, the findings may not generalize to female recreational athletes. In future studies, we wish to have more balanced gender participation.

Based on our findings, we will co-design personal informatics systems with recreational athletes, and examine how they are used by athletes to meet personal goals. This would allow us to further refine our findings and design opportunities.

\section{REFERENCES}

[1] 2015. Sports and Health in America. https://media.npr.org/documents/2015/june/ sportsandhealthpoll.pdf

[2] 2019. The Future of Biosensing Wearables. https://rockhealth.com/reports/ the-future-of-biosensing-wearables/ Accessed: January, 2019.

[3] Mira Alhonsuo, Jenni Hapuli, Lasse Virtanen, Ashley Colley, and Jonna Häkkilä. 2015. Concepting wearables for ice-hockey youth. In Proceedings of the 17th International Conference on Human-Computer Interaction with Mobile Devices and Services Adjunct. ACM, 944-946.

[4] Patrick Carrington, Kevin Chang, Helena Mentis, and Amy Hurst. 2015. But, I don't take steps: Examining the Inaccessibility of Fitness Trackers for Wheelchair Athletes. In Proceedings of the 17th International ACM SIGACCESS Conference on Computers \& Accessibility. ACM, 193-201.

[5] Eun Kyoung Choe, Nicole B Lee, Bongshin Lee, Wanda Pratt, and Julie A Kientz. 2014. Understanding quantified-selfers' practices in collecting and exploring personal data. In Proceedings of the SIGCHI Conference on Human Factors in Computing Systems. ACM, 1143-1152.

[6] James Clawson, Jessica A Pater, Andrew D Miller, Elizabeth D Mynatt, and Lena Mamykina. 2015. No longer wearing: investigating the abandonment of personal health-tracking technologies on craigslist. In Proceedings of the 2015 ACM International foint Conference on Pervasive and Ubiquitous Computing. ACM, 647-658.

[7] Sunny Consolvo, Katherine Everitt, Ian Smith, and James A Landay. 2006. Design requirements for technologies that encourage physical activity. In Proceedings of the SIGCHI conference on Human Factors in computing systems. ACM, 457-466.
[8] Sunny Consolvo, Predrag Klasnja, David W McDonald, Daniel Avrahami, Jon Froehlich, Louis LeGrand, Ryan Libby, Keith Mosher, and James A Landay. 2008. Flowers or a robot army?: encouraging awareness \& activity with personal, mobile displays. In Proceedings of the 10th international conference on Ubiquitous computing. ACM, 54-63.

[9] Daniel A Epstein, Monica Caraway, Chuck Johnston, An Ping, James Fogarty, and Sean A Munson. 2016. Beyond abandonment to next steps: understanding and designing for life after personal informatics tool use. In Proceedings of the 2016 CHI Conference on Human Factors in Computing Systems. ACM, 1109-1113.

[10] Daniel A Epstein, An Ping, James Fogarty, and Sean A Munson. 2015. A lived informatics model of personal informatics. In Proceedings of the 2015 ACM International foint Conference on Pervasive and Ubiquitous Computing. ACM, 731-742.

[11] Thomas Fritz, Elaine M Huang, Gail C Murphy, and Thomas Zimmermann. 2014. Persuasive technology in the real world: a study of long-term use of activity sensing devices for fitness. In Proceedings of the SIGCHI Conference on Human Factors in Computing Systems. ACM, 487-496.

[12] Francine Gemperle, Chris Kasabach, John Stivoric, Malcolm Bauer, and Richard Martin. 1998. Design for wearability. In digest of papers. Second international symposium on wearable computers (cat. No. 98EX215). IEEE, 116-122.

[13] Hayati Havlucu, Idil Bostan, Aykut Coskun, and Öuzhan Özcan. 2017. Understanding the Lonesome Tennis Players: Insights for Future Wearables. In Proceedings of the 2017 CHI Conference Extended Abstracts on Human Factors in Computing Systems. ACM, 1678-1685.

[14] HM Sajjad Hossain, Md Abdullah Al Hafiz Khan, and Nirmalya Roy. 2017. SoccerMate: A personal soccer attribute profiler using wearables. In 2017 IEEE International Conference on Pervasive Computing and Communications Workshops (PerCom Workshops). IEEE, 164-169.

[15] Brad Humphreys, Jane Ruseski, et al. 2008. The size and scope of the sports industry in the United States. In IASE Conference Papers, Vol. 833. International Association of Sports Economists.

[16] John M Jakicic, Kelliann K Davis, Renee J Rogers, Wendy C King, Marsha D Marcus, Diane Helsel, Amy D Rickman, Abdus S Wahed, and Steven H Belle. 2016. Effect of wearable technology combined with a lifestyle intervention on long-term weight loss: the IDEA randomized clinical trial. Fama 316, 11 (2016), $1161-1171$

[17] Amanda Lazar, Christian Koehler, Joshua Tanenbaum, and David H. Nguyen. 2015. Why We Use and Abandon Smart Devices. In Proceedings of the 2015 ACM International foint Conference on Pervasive and Ubiquitous Computing (UbiComp '15). ACM, New York, NY, USA, 635-646. https://doi.org/10.1145/2750858.2804288

[18] Ian Li, Anind Dey, and Jodi Forlizzi. 2010. A Stage-based Model of Personal Informatics Systems. In Proceedings of the SIGCHI Conference on Human Factors in Computing Systems (CHI '10). ACM, New York, NY, USA, 557-566. https: //doi.org/10.1145/1753326.1753409

[19] Brian Y. Lim, Aubrey Shick, Chris Harrison, and Scott E. Hudson. 2011. Pediluma: Motivating Physical Activity Through Contextual Information and Social Influence. In Proceedings of the Fifth International Conference on Tangible, Embedded, and Embodied Interaction (TEI '11). ACM, New York, NY, USA, 173-180. https://doi.org/10.1145/1935701.1935736

[20] Le Nguyen Ngu Nguyen, Daniel Rodríguez-Martín, Andreu Català, Carlos PérezLópez, Albert Samà, and Andrea Cavallaro. 2015. Basketball activity recognition using wearable inertial measurement units. In Proceedings of the XVI international conference on Human Computer Interaction. ACM, 60.

[21] Stina Nylander, Mattias Jacobsson, and Jakob Tholander. 2014. Runright: Realtime Visual and Audio Feedback on Running. In CHI '14 Extended Abstracts on Human Factors in Computing Systems (CHI EA '14). ACM, New York, NY, USA, 583-586. https://doi.org/10.1145/2559206.2574806

[22] Stina Nylander and Jakob Tholander. 2014. Designing for Movement: The Case of Sports. In Proceedings of the 2014 International Workshop on Movement and Computing (MOCO '14). ACM, New York, NY, USA, Article 130, 6 pages. https: //doi.org/10.1145/2617995.2618018

[23] Juho Rantakari, Virve Inget, Ashley Colley, and Jonna Häkkilä. 2016. Charting Design Preferences on Wellness Wearables. In Proceedings of the 7th Augmented Human International Conference 2016 ( $A H$ '16). ACM, New York, NY, USA, Article 28, 4 pages. https://doi.org/10.1145/2875194.2875231

[24] John Rooksby, Mattias Rost, Alistair Morrison, and Matthew Chalmers. 2014. Personal Tracking As Lived Informatics. In Proceedings of the SIGCHI Conference on Human Factors in Computing Systems (CHI '14). ACM, New York, NY, USA, 1163-1172. https://doi.org/10.1145/2556288.2557039

[25] Dominik Schuldhaus, Carolin Jakob, Constantin Zwick, Harald Koerger, and Bjoern M Eskofier. 2016. Your personal movie producer: generating highlight videos in soccer using wearables. In Proceedings of the 2016 ACM International Symposium on Wearable Computers. ACM, 80-83.

[26] Jakob Tholander and Stina Nylander. 2015. Snot, sweat, pain, mud, and snow: performance and experience in the use of sports watches. In Proceedings of the 33rd Annual ACM Conference on Human Factors in Computing Systems. ACM, 2913-2922. 